\title{
Prevention of Trauma and Stressor-Related Disorders: A Review
}

\author{
Jonathon R Howlett ${ }^{1}$ and Murray B Stein ${ }^{\star, 1,2,3}$ \\ ${ }^{1}$ Department of Psychiatry, University of California San Diego, La Jolla, CA, USA; ${ }^{2}$ Department of Family Medicine and Public \\ Health, University of California San Diego, La Jolla, CA, USA; ${ }^{3}$ VA San Diego Healthcare System, San Diego, CA, USA
}

Posttraumatic stress disorder (PTSD) is a common, frequently chronic, and disabling condition which, along with acute stress disorder (ASD), is categorized as a trauma- and stressor-related disorder by the DSM-5. These disorders are unique in requiring exposure to a severe stressor, which implies that potential sufferers could be identified and helped before developing a disorder. Research on prevention strategies for stress-related disorders has taken a number of avenues, including intervention before and after trauma and the use of both psychosocial and somatic approaches. Despite advances in neurobiological understanding of response to trauma, clinical evidence for preventive interventions remains sparse. This review provides an overview of prevention approaches and summarizes the existing literature on prevention of ASD and PTSD, including clinical and preclinical studies. Given the potential benefits to trauma survivors and society, the development of effective preventive interventions should be given greater priority. Resources should be directed to adequately test promising interventions in clinical trials, and research should be conducted according to translational research principles in which preclinical research informs the design of clinical studies.

Neuropsychopharmacology Reviews (2016) 41, 357-369; doi: I0.1038/npp.20I5.26I; published online 23 September 2015

\section{INTRODUCTION}

Posttraumatic stress disorder (PTSD) is a common, frequently chronic, and disabling condition that represents a major public health burden on par with other severe mental illnesses (Kessler, 2000). PTSD, along with acute stress disorder (ASD), is classified as a trauma- and stressorrelated disorder by the fifth edition of the Diagnostic and Statistical Manual of Mental Disorders (DSM-5) (American Psychiatric Association, 2013). These disorders are unique in that diagnosis requires exposure to an extreme stressor such as threat of death, serious injury, or sexual violence. In the presence of such a clearly identifiable inciting event, clinicians may be positioned to intervene to prevent these disorders before they take hold. In light of the chronicity of PTSD and its associated suffering and impairment, successful prevention would clearly represent a major breakthrough benefiting trauma survivors and society. This opportunity has prompted research developing both psychosocial and pharmacologic approaches to prevent PTSD. Advances in neurobiological understanding could

\footnotetext{
${ }^{*}$ Correspondence: Dr M Stein, Department of Psychiatry and Department of Family Medicine and Public Health, University of California San Diego, VA San Diego Healthcare System, 9500 Gilman Drive, La Jolla, CA 92093-0855, USA, Tel: +1 858534 6451, Fax: +1 858534 6460, E-mail: mstein@ucsd.edu

Received 30 March 2015; revised 9 July 2015; accepted 11 August 2015; accepted article preview online 28 August 2015
}

engender tools both to identify trauma survivors at high risk of PTSD and to intervene before symptoms begin. However, despite the progress, clinical evidence for preventive interventions is distressingly underdeveloped (Stein and Lang, 2013). More efforts to leverage preclinical models to develop and test new prevention strategies are sorely needed.

ASD and PTSD are closely related. Many ASD patients later develop PTSD, although some individuals who go on to have PTSD do not meet ASD criteria in the aftermath of trauma (Bryant, 2011). In addition to exposure to a stressor, both disorders require additional symptoms, which may include intrusion, avoidance, changes in mood and cognition, and changes in arousal and reactivity. ASD requires symptoms to occur for at least 3 days and less than 1 month after the traumatic event, while PTSD requires symptoms to persist for at least 1 month. Studies have estimated that more than $80 \%$ of adults in the United States experience traumatic events that would qualify for ASD or PTSD diagnosis (Breslau, 2012). Categories of trauma that may result in ASD and PTSD include assaultive violence including military combat and sexual assault, other injuries such as motor vehicle accidents, and witnessing or learning of a sudden death or serious injury in another person. As a whole, assaultive violence is more likely to result in ASD and PTSD than other types of trauma and carries about a $20 \%$ risk for ASD and PTSD (Brewin et al, 2014). Overall, PTSD has an estimated $6.8 \%$ lifetime prevalence in the United States 
(Kessler et al, 2005). Importantly, efforts are underway to expand understanding of individual variability in posttraumatic responses based on different patterns of symptoms, time courses of symptoms, or underlying biological differences (Insel et al, 2010; Zoladz and Diamond, 2013). These investigations could lead to alternative classification systems beyond the DSM categories of ASD and PTSD.

In this review, we summarize research on the prevention of ASD and PTSD. We begin with a general overview and classification of approaches to prevention, along with special considerations for prevention of stress-related disorders. We consider alternative classification systems that account for variability in posttraumatic responses not captured by categorical DSM diagnoses, which may prove useful in individualized preventive interventions. We then briefly discuss primary prevention approaches including prevention of traumatic events as well as pretrauma interventions to improve resilience in the face of trauma. Then, we examine secondary prevention, including the targeting of interventions, psychosocial approaches, and somatic approaches. We conclude by discussing future directions in this important research area.

When the evidence for a particular intervention has been covered in detail in previous reviews, we will summarize the overall evidence rather than enumerate all studies. Interested readers are referred to the appropriate reviews for more detail. For example, two psychosocial interventions, psychological debriefing (PD) and cognitive behavioral therapy (CBT), have been the subject of numerous studies previously discussed in comprehensive reviews, and we do not describe each study in detail. In contrast, a larger number of separate somatic interventions have been tested in one or a handful of studies. For this reason, we discuss somatic approaches at somewhat greater length overall than psychosocial approaches, which is a consequence of our aim to address gaps in the current literature and maximize the overall utility of this review.

\section{OVERVIEW OF PREVENTION APPROACHES}

\section{Historical Systems for Classifying Prevention Strategies}

Discussions of disease prevention approaches need to contend with the question of what constitutes the scope of preventive interventions and how these interventions should be classified. We will describe two major historical systems for classifying disease prevention. The first system comprises primary, secondary, and tertiary prevention based on the stage of disease pathophysiology. The second system consists of universal, selective, and indicated prevention based on the population or group being targeted for prevention.

An early approach to classify preventive interventions was initially introduced in 1957 by the Commission on Chronic Illness and later broadened (Caplan, 1964; Commission on Chronic Illness, 1957). This classification scheme consists of primary, secondary, and tertiary prevention and is based on a mechanistic account in which biologic disease onset precedes presenting symptoms. Primary prevention occurs before the biologic onset of disease and seeks to reduce disease incidence. Primary prevention efforts could take the form of general health promotion (eg, promotion of good nutrition) or attempts to prevent a specific disease (eg, vaccines). Prevention efforts could also focus either on reducing exposure to environmental risk factors or on improving individuals' resilience in the face of risk factors (Rose, 1992). Secondary prevention occurs after a disease has originated but before it becomes symptomatic. Pre-symptomatic diseases can be identified by screening programs. Finally, tertiary prevention refers to prevention of further decline or disability after a disease has already manifested, and overlaps substantially with treatment.

Gordon (1983) advanced a critique of the distinction between primary and secondary prevention on the basis that this distinction requires a mechanistic biologic understanding of disease and that it is often difficult to identify a specific origin in multifactorial, complex diseases that may have several preexisting risk factors. He proposed an alternative system in which prevention approaches are classified according to the population or group to which they are targeted. Universal prevention is targeted toward the entire population. Selective prevention is targeted toward a subgroup of the population, which has been shown to be at higher risk for the disorder. Subgroups could be distinguished by age, gender, occupation, family history, or other characteristics. Finally, indicated prevention is targeted toward individuals with a condition or abnormality that identifies them as being at high risk for disease, although they remain asymptomatic.

\section{Special Considerations for Prevention of Trauma- and Stressor-Related Disorders}

There are several special definitional considerations when discussing classification of preventive interventions for stress-related diseases (American Psychiatric Association, 2013). First, both ASD and PTSD require exposure to one or more traumas. Second, ASD and PTSD are defined in part according to the duration of symptoms after trauma exposure. ASD can be diagnosed when the duration of the disturbance is 3 days to 1 month after a trauma, while PTSD requires persistence of symptoms for more than 1 month. Finally, like other mental illnesses, ASD and PTSD are defined according to a set of symptoms, and the presence of asymptomatic disease might be considered a contradiction in terms (unlike for many physical health problems). The definitions of these disorders therefore present a challenge for the application of the distinction between primary and secondary prevention. Prior approaches to the prevention of stress-related diseases have considered any intervention before trauma exposure to be primary prevention, while any intervention after trauma exposure but before development of PTSD (ie, before 1 month of symptoms) to be 
secondary prevention (Skeffington et al, 2013; Stein and Lang, 2013). For the purposes of this review, we will adopt the same convention. Primary prevention includes any intervention designed to prevent trauma exposure as well as attempts to improve resilience before a trauma. Primary prevention approaches could be either universal or selective. Secondary prevention could occur for asymptomatic individuals, but also for those who have symptoms but do not yet meet criteria for PTSD (eg, those having symptoms for less than 1 month). Secondary prevention could be targeted toward individuals meeting criteria for ASD. The distinction between ASD treatment and PTSD prevention may not always be clear, but we will focus on studies that assessed subsequent development of PTSD rather than merely change in ASD symptoms. Tertiary prevention (interventions after onset of PTSD which overlap with PTSD treatment) will not be considered in this review.

\section{Categorical and Dimensional Approaches to Posttraumatic Symptoms}

Recent perspectives on mental disorders including trauma and stressor-related disorders have emphasized the importance of individual heterogeneity within DSM diagnoses in terms of symptoms, time course, underlying biological dysfunctions, and other factors. In particular, patients meeting criteria for PTSD may differ in terms of underlying biology, symptom profile, course of illness, and response to interventions. To optimize prevention strategies for all trauma survivors, it may be necessary to account for variability that is not captured by a categorical DSM diagnosis. Recognizing this problem, the National Institute of Mental Health (NIMH) has launched the Research Domain Criteria (RDoC) project. $\mathrm{RDoC}$ aims to create a framework to develop improved classification schemes based on new pathophysiological findings, including discoveries in genomics and neuroscience, rather than solely based on symptoms (Insel et al, 2010). While a full overview of alternatives to the DSM classification scheme for trauma and stressor-related disorders is outside the scope of this review, we will briefly introduce a handful of approaches that may prove useful in developing and targeting preventive interventions.

Alternative approaches to classification of posttraumatic pathology have begun yielding insights that may be useful when developing and targeting preventive interventions. Such studies have used a number of statistical methods to identify patterns in posttraumatic symptoms, both in cross-sectional and in longitudinal designs. Confirmatory factor analysis (CFA) is used to uncover underlying factors that reflect the latent structure of diagnoses such as PTSD. Application of this method has yielded two prominent four-factor models of PTSD (King et al, 1998; Simms et al, 2002). This research influenced the change in criteria from DSM-IV (which includes three PTSD symptom clusters) to DSM-5 (which includes four) (American Psychiatric Association, 2000, 2013). However, recent competing models have also been proposed, ranging from two factors to seven factors (Forbes et al, 2015a; Zelazny and Simms, 2015).

Latent class analysis (LCA) is a method that uses observed symptoms to identify subgroups in a population, which are not directly observable. Unlike CFA, which identifies underlying dimensional factors, LCA can be used to assign individuals to discrete groups. LCA has been used to characterize PTSD presentation in terms of severity (Steenkamp et al, 2012), personality profiles of PTSD patients (Forbes et al, 2010), a dissociative subtype of PTSD (Wolf et al, 2012), PTSD comorbidity (Galatzer-Levy et al, 2013), and psychopathology after trauma (not limited to PTSD) (Forbes et al, 2015b). A related method, latent transition analysis (LTA), has been used to examine the movement of trauma-exposed individuals between subgroups over time (Forbes et al, 2015b).

Another type of method categorizes trauma-exposed individuals based on their trajectory of symptoms over time. Rather than characterizing the overall mean level of symptoms at each time point, these methods separate individuals into groups following different trajectories over time. For example, a 6-year study using latent growth mixture modeling (LGMM) identified five distinct trajectories of PTSD symptoms after injury, including resilient (representing a majority of individuals), chronic, recovery, worsening/recovery, and worsening (Bryant et al, 2015). Improved understanding of different time courses of symptoms after trauma could help elucidate the influence of preventive interventions on different individuals.

As reviewed above, new approaches to classification of posttraumatic symptoms may improve prevention efforts by tailoring interventions based on factors specific to an individual. Nevertheless, most research to date has relied on DSM criteria, and progress has been made in the identification and evaluation of preventive interventions using these criteria. For this reason, the majority of studies reviewed here use DSM criteria. Future research will likely evince an expansion of efforts to more fully account for variability in trauma-exposed individuals, potentially resulting in better preventive efficacy through targeted interventions.

\section{PRIMARY PREVENTION}

\section{Prevention of Traumatic Incidents}

One approach to prevention of stress-related disorders is the prevention of exposure to trauma. Traumatic events that can lead to PTSD include assaultive violence (including military combat trauma, rape, and physical assault), other injuries (including motor vehicle accidents and other accidents), and learning about sudden death or injuries of loved ones. Assaultive violence is more likely to result in PTSD than other traumas (Breslau, 2012). Efforts to prevent injuries and assaults include a large variety of public health and policy interventions, which are beyond the scope of this review. Injury prevention strategies include recall of defective motor 
vehicles, implementation of blood alcohol limits for drivers, and addition of separation barriers to freeways, among many others (Kraus et al, 1998). Interventions to reduce assaultive violence include educational programs, strengthening of community-based support services, laws limiting access to firearms, and attempts to improve identification of victims of violence by medical personnel to prevent further victimization (Rosenberg et al, 1998). Strategies to prevent trauma include universal measures and selective measures (such as those targeting low socio-economic status families).

\section{Pre-Trauma Resilience Building}

Hourani et al (2011) reviewed studies of predeployment interventions meant to mitigate the impact of combat-related stressors in military populations. Approaches included psychoeducation programs to educate military personnel about reactions to trauma as well as stress inoculation training, which aims to improve ability to cope with stressors. However, most studies were descriptive and did not include a control group for comparison of outcomes. A controlled, non-randomized, parallel group study compared mental health outcomes between military personnel deployed to Iraq who received pre-operational stress briefing and those who did not and found no difference in PTSD, other mental health disorders, or alcohol misuse (Sharpley et al, 2008).

Skeffington et al (2013) performed a review of primary prevention of PTSD, which was not restricted to studies in military populations. The literature was extremely limited, with only seven studies identified as meeting eligibility criteria. Studies of pretrauma psychoeducation were nonrandomized and were insufficient to make any determination concerning efficacy. A handful of studies examining interventions combining psychoeducation and skills building either used non-randomized design or did not measure PTSD symptoms at follow-up. Finally, two very small studies of police officers showed that stress management training could reduce physiologic arousal in response to a simulated stressor, but did not measure response to actual stressors and did not measure actual PTSD symptoms. Overall, the authors concluded that there is currently insufficient evidence for any primary prevention strategy for PTSD and that more research is needed in this area.

\section{SECONDARY PREVENTION}

\section{Targeting Interventions}

Overall, fewer than $10 \%$ of individuals exposed to a traumatic event go on to develop PTSD, although the rate for assaultive violence is approximately $20 \%$ and for rape is as high as 50\% (Breslau, 2012). If techniques are available to identify individuals at highest risk for developing PTSD after a trauma, then preventive interventions could be targeted to those individuals. There is an extensive body of research on risk factors for PTSD, and a full summary is outside the scope of this review. We will therefore only provide a very brief introduction to the topic. It should be emphasized that the risk factors reviewed here have poor sensitivity and specificity in identifying individuals that will develop PTSD, and as yet the only clinically useful factor in targeting intervention has been the emergence of symptoms (eg, ASD) in the aftermath of trauma. However, future risk prediction tools that incorporate an array of risk factors may be more successful in identifying individuals most likely to benefit from intervention.

Research has indicated that risk factors for PTSD include female gender, preexisting psychiatric disorders, family history of psychiatric disorders, lower IQ, personality traits such as neuroticism, and low social support (Breslau, 2012). In addition to these risk factors, a recent review also identified psychophysiological predictors of PTSD, most of which were related to arousal (DiGangi et al, 2013). These included startle reactivity, salivary elevations in the norepinephrine metabolite 3-methoxy-4-hydroxyphenylglycol (MHPG), alterations in the glucocorticoid system, attentional bias away from fearful faces, and reduced extinction of a conditioned emotional response. Research has established that the development of PTSD after trauma is moderately heritable, and a growing number of studies have attempted to identify genes associated with PTSD. Candidate gene studies, in which genes are selected for study based on biological pathways thought to have a role in PTSD, have identified a number of genes possibly associated with risk of PTSD. These include genes involved in the hypothalamicpituitary-adrenal (HPA) axis, the noradrenergic, serotonergic and dopaminergic systems, memory consolidation, and other biological systems (Almli et al, 2014; Logue et al, 2015). However, some of these candidate gene studies have failed to replicate. Another approach to identify genetic variants associated with PTSD is a genome-wide association study (GWAS), in which genetic variation is examined without any prior hypotheses about particular genes. To date, four GWAS of PTSD have been published, which have identified five loci associated with PTSD (Logue et al, 2015). It should be noted that each identified genetic variant likely accounts for only a small proportion of variance in the disorder (Nievergelt et al, 2015).

In addition to pre-trauma risk factors, risk for PTSD could also be stratified based on reactions in the immediate aftermath of trauma. Bryant (2011) reviewed 22 studies that assessed for ASD within 1 month of trauma and later assessed for PTSD. Positive predictive power was fairly high, with most studies finding that more than half of individuals who met ASD criteria went on to develop PTSD. However, sensitivity was low, as indicated by the fact that the majority of trauma survivors who went on to develop PTSD did not meet full ASD criteria. It should be noted that subsequent to this review, DSM-5 removed the requirement for dissociative symptoms for ASD diagnosis, thereby making the diagnosis less stringent (American Psychiatric Association, 2013). Several studies have shown that removal of the requirement for dissociative symptoms improves the sensitivity in 
predicting subsequent PTSD (Bryant, 2011). A recent study confirmed that ASD diagnosed based on DSM-5 criteria compared with DSM-IV has greatly improved sensitivity with comparable specificity in predicting subsequent PTSD (Bryant et al, 2014).

Research has suggested that measures of biological and cognitive mechanisms in the acute aftermath of trauma may offer more accurate prediction of PTSD than any constellation of acute symptoms (Bryant, 2003). Markers in the acute period after trauma that predict development of PTSD include elevated resting heart rate and respiration rate (Bryant et al, 2008), low plasma levels of gamma amino-butyric acid (GABA) (Vaiva et al, 2004), and maladaptive cognitive factors including negative appraisals (Dunmore et al, 2001).

As noted above, no individual risk factors for PTSD exhibit sufficient sensitivity and specificity to confidently identify individuals who will develop the disorder. An approach that may overcome this difficulty is the use of machine learning methods that integrate a number of clinical and biological risk factors to identify individuals at highest risk for developing the disorder. A recent study of 957 trauma survivors used machine learning methods to predict PTSD status at 15 months based on clinical and biological information collected within 10 days of trauma (Galatzer-Levy et al, 2014). The machine learning approach using a combination of factors outperformed ASD symptoms in predicting PTSD. In another study, machine learning methods were used to create a prediction algorithm for PTSD on the basis of retrospectively assessed risk factors (including trauma type, socio-demographics, prior history of trauma, and mental illnesses) for 9791 subjects (Kessler et al, 2014). Over 95\% of all PTSD cases were associated with the $10 \%$ of trauma exposures classified by the algorithm as having the highest PTSD risk. The authors note that the approach will need to be applied in a prospective study to validate the findings. Taken together, research on PTSD risk factors suggests that prediction tools may be developed to stratify risk for PTSD and allow for targeted secondary prevention strategies.

\section{Psychosocial Approaches}

A number of psychosocial interventions have been tested for prevention of PTSD. Approaches differ in their components and theoretical grounding, the number of sessions, timing of the intervention, and targeting (eg, whether they are delivered only to patients with ASD symptoms or to all trauma survivors). The earliest approaches tended to be single-session interventions, often delivered in group settings to all survivors of a trauma. Later approaches used multi-session interventions based on cognitive behavioral therapy (CBT) principles and frequently targeted toward patients with full or subclinical ASD. In addition to these approaches, a number of other interventions have also been studied. The evidence for several psychosocial approaches to PTSD prevention, including single session and CBT-based interventions, has been examined in depth in several reviews (Agorastos et al, 2011; Bryant, 2007; Forneris et al, 2013; Kearns et al, 2012; Rose et al, 2002). For this reason, we will summarize evidence for each approach but will not describe each study separately. Readers interested in a more comprehensive account of the evidence for these approaches are referred to the cited reviews.

Psychological debriefing (PD) is a single-session intervention provided to trauma survivors in the early aftermath of a traumatic incident. PD originated in the military, where commanders would meet with personnel after a battle to discuss their experiences with the intention of improving morale and preparing for future combat (Litz et al, 2002). There are several different forms of PD, with critical incident stress debriefing (CISD) being among the most widely used (Mitchell and Everly, 1993). CISD includes a number of components, including discussion of the traumatic event, sharing of thoughts and emotions about the event, and education about typical stress reactions and stress management strategies. While CISD was originally designed specifically for emergency service personnel and disaster workers, similar interventions have been used outside this context, such as in victims of violent crime (Rose et al, 1999). Despite widespread implementation, evidence has accumulated that $\mathrm{PD}$ is not an effective intervention in preventing PTSD. A systematic review of interventions to prevent PTSD by Forneris et al (2013) concluded that PD did not reduce PTSD incidence or symptom severity, while a Cochrane Review concluded that PD was either equivalent to or worse than control or psychoeducation in preventing or reducing PTSD severity (Rose et al, 2002). Overall, PD is not supported by the evidence as an intervention to prevent PTSD.

While single-session PD interventions do not appear effective at preventing PTSD, multiple session interventions using a CBT framework have received some support. These interventions are adapted from CBT approaches, which are known to be effective treatments for chronic PTSD (Harvey et al, 2003) and include psychoeducation, exposure, cognitive restructuring, and anxiety management (Bryant, 2007). These interventions occur in the first weeks or months after trauma and typically consist of 4-12 sessions (Kearns et al, 2012) with the most frequent length being about 5 sessions (Bryant, 2007). The largest trial is the Jerusalem Trauma Outreach and Prevention Study, which randomized a total of 242 trauma survivors with early PTSD symptoms to either 12 sessions of one of two forms of CBT (prolonged exposure or cognitive therapy), the selective serotonin reuptake inhibitor (SSRI) escitalopram or placebo, or to a waiting list (Shalev et al, 2012). Results indicated that both prolonged exposure and cognitive therapy were effective in preventing PTSD at 5 months (with approximately $20 \%$ of subjects meeting PTSD criteria compared with nearly $60 \%$ on the waiting list). Both therapy groups outperformed escitalopram, which was equivalent to placebo. A later study reanalyzed the same data using LGMM to assess different symptom trajectories of subgroups of patients (Galatzer-Levy et al, 2013). The 
authors identified three subgroups based on symptom trajectories: rapid remitting, slow remitting, and nonremitting. Furthermore, the authors concluded that CBT accelerated recovery of the slow remitting group but did not affect the other two groups. The use of LGMM and similar methods may help clarify the influence of preventive interventions on specific subgroups of patients and may ultimately help target treatment to those most likely to benefit.

In addition to the Jerusalem Trauma Outreach and Prevention Study, smaller trials have also found evidence for the efficacy of CBT in patients who exhibit early symptoms. These studies are summarized in several reviews, which have concluded that $\mathrm{CBT}$ is effective at reducing subsequent PTSD incidence or severity in individuals with ASD (Agorastos et al, 2011; Bryant, 2007; Kearns et al, 2012). However, in another review, Forneris et al (2013) stated that the evidence in support of early CBT remains tentative due to limited evidence. A Cochrane Review has also concluded that CBT is an effective treatment for ASD (Roberts et al, 2010).

The utility of CBT in trauma survivors who do not meet criteria for ASD is less clear. Studies of psychological interventions administered to all trauma survivors irrespective of symptoms have typically utilized counseling interventions rather than CBT. A meta-analysis including eight such studies found no significant overall effect (Roberts et al, 2009). The same meta-analysis found that CBT interventions were effective at preventing PTSD when targeted to individuals exhibiting traumatic stress symptoms, with the greatest effect for individuals meeting full criteria for ASD or acute PTSD. A more recent RCT compared a three-session exposure-based intervention beginning in the emergency department with assessment only in 137 adult trauma survivors (Rothbaum et al, 2012). The intervention was delivered to all trauma survivors regardless of symptoms and was initiated as early as possible based on preclinical evidence suggesting that early extinction training can block fear memory consolidation. Participants receiving the intervention had lower levels of posttraumatic stress reactions at 4- and 12-week follow-up. Two follow-up studies by the same group examined predictors of PTSD symptoms and response to the exposure-based intervention. The first study found that dissociation after trauma predicted poor response to the intervention and also predicted greater PTSD symptoms in the control group (Price et al, 2014). The second study found that a group of genetic variants related to the stress response appeared to confer risk of increased PTSD symptoms in control subjects, but that this increased risk appeared to be mitigated by the exposure intervention (Rothbaum et al, 2014). More studies of CBT-based interventions in trauma survivors irrespective of symptoms are needed to draw definitive conclusions about their utility in this population. Overall, the balance of evidence indicates that CBT is effective at preventing PTSD for individuals with ASD, and possibly those with sub-threshold or no symptoms, although more research is needed.
Another multi-session intervention that is designed specifically to prevent PTSD in trauma-exposed youth is the Child and Family Traumatic Stress Intervention (CFTSI). In this approach, both child and caregiver undergo four sessions designed to improve caregiver support for the child and to improve coping behaviors. A study was performed in which 106 trauma-exposed youth reporting at least one new distressing posttraumatic symptom were randomized to receive CFTSI or a four-session supportive control intervention (Berkowitz et al, 2011). At 3 months, the CFTSI group had a lower rate of both full and partial PTSD diagnoses.

Another model for PTSD prevention is a collaborative care approach, in which trauma survivors are assigned a case manager and then possibly referred for CBT, pharmacologic management, or a combination on an individual basis depending on symptoms and patient preferences. One RCT has tested this approach in 207 acutely injured hospitalized patients and found that those receiving the collaborative care intervention had reduced PTSD symptoms over the year after injury compared with those who received care as usual (Zatzick et al, 2013). While more studies are needed to test this approach to PTSD prevention, the use of case management to identify individual trauma survivors who require psychological or pharmacologic interventions may represent a promising avenue to structure prevention efforts.

Several other psychosocial approaches to PTSD prevention have been tested, mostly in small studies. A memory structuring intervention that aims to shift processing of traumatic memories from uncontrolled affective processing to controlled cognitive and linguistic processing has shown mixed results in two small studies (Gidron et al, 2001, 2007). A limited body of evidence examining psychoeducation approaches does not currently support the efficacy of these interventions in preventing PTSD (Wessely et al, 2008). Battlemind, an intervention for military personnel exposed to combat trauma which was adapted from PD, found preliminary support from a study randomized at the platoon level but did not show an effect in another study (Adler et al, 2011; Mulligan et al, 2012). A study using an informational video designed for sexual assault survivors found reduced PTSD symptoms at 6 weeks only for women with a prior history of sexual assault, with the effect disappearing at 6 months (Resnick et al, 2007). Overall, more research is needed on these interventions before they can be considered effective at preventing PTSD.

In summary, the psychosocial intervention for PTSD prevention with the most evidence is brief CBT. Most studies have examined this approach in individuals either meeting ASD criteria or with subclinical traumatic symptoms. Due to the lack of evidence, no firm conclusions can be drawn about the efficacy of brief CBT in trauma survivors without significant symptoms. There is some evidence for a collaborative care approach in which acutely injured patients are assigned a case manager who can then refer patients for psychotherapy or medication management based on individual needs. However, more research is needed on this approach. PD appears to be ineffective in preventing PTSD 
among trauma survivors. Finally, a number of other interventions have been developed, but currently lack enough evidence to conclude they are effective in preventing PTSD. More research is needed, both to further test various preventive interventions and to identify which individual trauma survivors are most likely to benefit from preventive interventions.

\section{Somatic Approaches}

Safe and effective pharmacologic interventions to prevent stress-related disorders after trauma could be widely implemented and result in a substantial public health impact. However, the current evidence base for such approaches remains very small. Only a handful of interventions have been tested in RCTs, and many of these clinical studies have been small. Most approaches tested in RCTs have not found clear and consistent support. Some interventions have been tested in observational studies but not yet examined in RCTs. Finally, rodent studies have identified a number of modulators of the stress response and memory consolidation which may be useful for PTSD prevention, but have not been tested in humans.

At this time, the pharmacologic intervention with the strongest evidence for PTSD prevention is glucocorticoid administration. Endogenous glucocorticoids function to orchestrate physiologic and behavioral responses to maintain homeostasis in the context of stressful events, and inadequate release of glucocorticoids in response to a stressor may interfere with information processing and disrupt long-term memory integration (Zohar et al, 2011). Human studies have found that lower cortisol levels immediately after a trauma predict future PTSD symptoms (Delahanty et al, 2000; McFarlane et al, 1997). Animal research has shown that rats with a hypoactive HPA axis are more prone to behavioral disruption after a stressor than rats with a hyperactive HPA axis, and also that administration of a glucocorticoid after a stressor reduces PTSD-like behaviors (Cohen et al, 2008).

Two studies in humans examined the effect of the administration of stress doses of hydrocortisone in critically ill patients or patients undergoing major surgery. The first study recruited 20 subjects from a larger RCT in which patients with septic shock were administered hydrocortisone or a placebo (Schelling et al, 2001). Patients who received hydrocortisone were less likely to meet criteria for PTSD at follow-up (median 31 months after hospital discharge). The second study was a RCT in which 91 patients undergoing cardiac surgery received either stress doses of hydrocortisone or standard treatment and found that patients receiving hydrocortisone had lower PTSD symptoms at 6-month follow-up (Schelling et al, 2004). These results are also supported by a retrospective case-control study in which patients with septic shock who had received high-dose hydrocortisone had lower incidence of PTSD several years after ICU discharge than those who had not (Schelling et al, 1999). These studies suggest that stress dose glucocorticoids as an intervention to prevent PTSD in individuals undergoing critical illness or major surgery deserves more study, but by themselves cannot be generalized to contexts other than critical illness or major surgery.

Two RCTs have tested hydrocortisone in adults exposed to traumatic events who are not critically ill. In one trial, 25 patients with symptoms of ASD received either a single high dose of hydrocortisone or placebo within $6 \mathrm{~h}$ of exposure to a traumatic event (Zohar et al, 2011). Patients receiving hydrocortisone had significantly lower PTSD symptoms at 3 months. In another trial, 64 traumatic injury patients received a 10-day course of low-dose hydrocortisone or placebo initiated within $12 \mathrm{~h}$ of trauma, and those receiving hydrocortisone had lower PTSD symptoms at 3 months (Delahanty et al, 2013). Overall, while glucocorticoids for PTSD prevention need more study, preliminary studies have shown promise that they may be effective for some trauma survivors.

Another pharmacologic intervention that has been tested for prevention of stress-related disorders is propranolol, a beta-adrenergic antagonist. Preclinical studies have shown that the adrenergic system is involved in memory enhancement in the context of stress and hyperarousal, and that administration of propranolol can block memory consolidation after a stressful learning task (Cahill et al, 2000; Southwick et al, 1999). An early small non-randomized study that compared 11 trauma survivors with tachycardia who received a course of propranolol with eight similar patients who refused propranolol found that patients receiving propranolol exhibited lower levels of PTSD symptoms at 2 months (Vaiva et al, 2003). However, several RCTs have not found an effect of propranolol in preventing PTSD. Three RCTs consisted of adults who had experienced a traumatic event and presented to an emergency department or were admitted to a trauma center. In two studies, propranolol was compared with placebo (Hoge et al, 2012; Pitman et al, 2002), and in another study propranolol was compared with both placebo and gabapentin (Stein et al, 2007). None of these trials found significant differences in PTSD incidence or PTSD symptoms between patients receiving propranolol or placebo. However, Pitman et al (2002) did find reduced physiologic reactivity to trauma-related imagery at 3 months in those who had received propranolol. Another RCT compared a course of propranolol with placebo in pediatric injury patients and also failed to find an overall difference in PTSD symptoms, although girls receiving propranolol actually exhibited higher PTSD symptoms than those receiving placebo (Nugent et al, 2010). A retrospective chart review of 363 pediatric burn patients who had received propranolol or placebo as part of an earlier RCT found no significant difference in rates of ASD between patients receiving propranolol vs placebo (Sharp et al, 2010). Differences in study population or time of administration may influence the outcome of studies of propranolol. For example, Pitman et al administered propranolol within $6 \mathrm{~h}$ of trauma, while other studies allowed for later administration. It should also be noted that in some 
trials only a relatively small portion of eligible subjects consented to participate in medication studies. Overall, current evidence does not support propranolol as an intervention to prevent stress-related disorders.

Several SSRIs have shown efficacy in the treatment of chronic PTSD (Jonas et al, 2013). Two RCTs have examined the efficacy of early initiation of an SSRI in preventing PTSD. As part of the Jerusalem Trauma Outreach and Prevention Study (discussed above in the context of CBT interventions), 46 adult trauma survivors with PTSD symptoms were randomized to receive escitalopram or placebo beginning approximately 20 days after trauma (Shalev et al, 2012). There were no differences in PTSD symptoms at 5-month and 9-month follow-up. As noted above, prolonged exposure and cognitive therapy (two forms of CBT) were found to be effective and outperformed escitalopram in the same trial. Another RCT administered sertraline or placebo to 26 child and adolescent burn patients and found greater reduction in PTSD symptoms at 24 weeks among those receiving sertraline as assessed by parent report, but not by child report (Stoddard et al, 2011). Based on these two small samples, evidence for SSRIs in preventing PTSD remains equivocal.

Benzodiazepines are effective for anxiety and are frequently prescribed to chronic PTSD patients, although evidence does not support their efficacy for this disorder and there are a number of potential adverse reactions in this population (Lund et al, 2012). Preclinical studies have found that pre-administration of benzodiazepines before exposure to inescapable stress prevents the release of norepinephrine from the locus coeruleus, which has been posited to contribute to the development of PTSD (Bremner et al, 1993). Benzodiazepines also reduce arousal, decrease distress, and inhibit memory consolidation (Barbee, 1993). These findings led to the idea that benzodiazepines may prevent PTSD if administered in the acute aftermath of a trauma. However, two small clinical studies do not support benzodiazepines in preventing PTSD. A non-randomized study compared 13 trauma survivors exhibiting symptoms such as anxiety or insomnia who received a course of either alprazolam or clonazepam with 13 matched controls who did not receive a benzodiazepine, but found no difference in PTSD symptoms at 1-month and 6-month follow-up (Gelpin et al, 1996). At 6 months, 9 of 13 benzodiazepine subjects and 3 of 13 controls met PTSD criteria. Another study randomized 22 survivors of life-threatening injuries with PTSD symptoms to receive a 7-day course of temazepam or placebo and found no difference in PTSD severity at 6-week follow-up (Mellman et al, 2002). The risks of benzodiazepines should also be considered, given the potential for tolerance and dependency and concern for increased morbidity and mortality (Ashton, 2005; Kripke, 2009). Benzodiazepines are often used to treat insomnia, a common PTSD symptom, but are not recommended for long-term insomnia treatment (Riemann et al, 2015). Overall, there is currently no clinical evidence for the efficacy of benzodiazepines for the prevention of PTSD.
Several other pharmacologic interventions for PTSD prevention have been tested in prospective clinical trials. Trauma survivors treated with gabapentin, an anticonvulsant with some evidence as an anxiolytic, did not differ in PTSD symptoms from patients treatment with placebo or propranolol in an RCT (Stein et al, 2007). A study in which 137 adult patients requiring mechanical ventilation were randomized to receive light sedation or deep sedation found no significant differences in PTSD symptoms at 4 weeks (Treggiari et al, 2009). An open label study tested prevention of PTSD with omega-3 fatty acids, which have been shown in animal models to promote hippocampal neurogenesis, a process that may facilitate clearance of fear memories and thereby attenuate the development of PTSD (Kawakita et al, 2006; Kitamura et al, 2009). Eleven survivors of accidental injury were given a course of omega- 3 fatty acid supplementation, and one patient met criteria for PTSD at 12-week follow-up, although there was no control group for comparison (Matsuoka et al, 2011). Overall, current evidence is not adequate to support any of these interventions for the prevention of PTSD.

Several medications, including opioids, ketamine, and albuterol, have shown a possible connection with lower likelihood of PTSD in observational studies, but have not yet been tested in clinical trials. Preclinical evidence suggests that opioids may impair fear conditioning in the amygdala and could thereby reduce the likelihood of PTSD (Good and Westbrook, 1995). Two prospective observational studies have examined the correlation between morphine administration and subsequent PTSD in children with burns. The first study found a significant correlation between morphine dose in the hospital and decrease in PTSD symptoms over 6 months (Saxe et al, 2001). The second study, which had only 11 completers, found a nonsignificant correlation between morphine dose in the hospital and decrease in PTSD symptoms over 6 months, but a significant correlation between morphine dose and decrease in the arousal cluster of symptoms (Stoddard et al, 2009). Two chart reviews have examined the association between morphine and subsequent PTSD symptoms in adult trauma survivors. One study compared acute morphine dose in traumatic injury patients who later developed PTSD with those who did not and found that PTSD patients had received significantly lower doses of morphine (Bryant et al, 2009). A second study compared injured military personnel who received a diagnosis of PTSD with those who did not in terms of early administration of morphine and found that a lower proportion of those with PTSD had received morphine (Holbrook et al, 2010). While several observational studies have found evidence that administration of morphine after a trauma is associated with lower likelihood of developing PTSD, this has not been tested in RCTs and needs further study before a causal link can be confirmed.

Ketamine, an NMDA receptor antagonist used for anesthesia and analgesia, appears to have rapid antidepressant properties and may be beneficial for chronic PTSD 
(Rasmussen, 2015). However, observational studies examining a possible link between ketamine administration and subsequent rates of PTSD have shown mixed results. Preclinical research is equivocal, with evidence showing that ketamine has anxiolytic effects but another study showing that administration of ketamine after a stressor does not prevent behavioral disruption (Juven-Wetzler et al, 2014; Zhang et al, 2014). A retrospective study of 56 injured accident victims found PTSD symptoms approximately 1 year post-accident were higher in those who had received (S)-ketamine during ambulance transportation, but were equal in those receiving racemic ketamine or opioids (Schönenberg et al, 2005). A chart review of 147 military service members who underwent surgery after a burn found that prevalence of subsequent PTSD was lower among those who had received intraoperative ketamine than those who had not (McGhee et al, 2008). However, a subsequent retrospective study by the same group that included 289 burned service members found no difference in incidence of PTSD among those who had received ketamine compared with those who had not (McGhee et al, 2014). It should be noted that intraoperative ketamine may or may not have similar effects to subanesthetic doses of ketamine administered to a conscious patient. More evidence is needed to resolve the inconsistent findings on the relationship between ketamine administration and subsequent PTSD.

Albuterol is a beta adrenergic agonist that is used for asthma, but has also been shown in animal models to impair avoidance learning, which is thought to be related to fear conditioning (Elías et al, 2004). An observational study assessed 38 motor vehicle accident survivors who had received albuterol after the accident (for asthma attacks or respiratory insufficiency) and 337 who did not receive albuterol (Kobayashi et al, 2011). At 6 weeks and 1 year after the accident, patients who had received albuterol exhibited lower levels of PTSD symptoms than those who had not. No RCTs have tested the efficacy of albuterol for PTSD prevention.

A number of approaches to PTSD prevention have shown promise in preclinical studies but have not yet been tested in clinical trials. Some studies have focused on neuropeptide systems, which are involved in regulating anxiety and the stress response. Neuropeptide Y (NPY), a regulator of the HPA axis and noradrenergic system that alters stress-related behaviors, was shown in an animal model to prevent anxious behaviors when administered intranasally after exposure to a stressor (Sabban et al, 2014). Oxytocin, another neuropeptide that is involved in social bonding and the attenuation of anxiety in response to stress, was found to decrease subsequent anxiety-related behaviors when administered after a stressor in rats (Cohen et al, 2010). Nociceptin is an opioid-related neuropeptide, which has receptors expressed in the central amygdala. A study found that an agonist of the nociceptin receptor (NOP-R) interfered with fear memory consolidation after a severe stressor in mice (Andero et al, 2013). Corticotropin-releasing factor (CRF) is a peptide hormone that has a central role in activating the
HPA axis in response to stress. An animal study found that blockade of the CRF1 receptor prevents initiation and consolidation of anxiety-like behavior after a severe stressor (Adamec et al, 2010).

Other neurotransmitter systems have also been targeted in preclinical studies of prevention strategies for PTSD. The cannabinoid system is thought to be an important regulator of anxiety, stress, and emotional learning, and a rat study found that administration of a cannabinoid receptor agonist after a trauma prevented trauma-induced alterations in anxiety behaviors (Ganon-Elazar and Akirav, 2012). Adenosine is a nucleoside that has been found to decrease activity in the amygdala and noradrenergic system, exert anxiolytic effects, and to be neuroprotective. A mouse study found that administration of an adenosine derivative after trauma exposure alleviated anxious behaviors and memory deficits and also prevented cell death in the hippocampus (Huang et al, 2014).

Other strategies tested in preclinical studies do not target specific neurotransmitter systems but instead modulate immune responses to stress or memory consolidation processes. Based on evidence that T-cell recruitment to the brain may help maintain brain functions and improve coping in the context of stress, mice were immunized with a central nervous system-related peptide before exposure to a stressor, and were found to develop reduced stressorrelated anxiety and acoustic startle response (Lewitus et al, 2008). Microinjection of anisomycin, a protein synthesis inhibitor, after stress exposure reduced subsequent anxiety-like and avoidant behavior, presumably by disrupting the process of traumatic memory consolidation (Cohen et al, 2006). Finally, a rat study found that sleep deprivation after exposure to a stressor ameliorated long-term PTSD-like behavioral disruptions, possibly by interfering with traumatic memory consolidation (Cohen et al, 2012).

In summary, no pharmacologic interventions to prevent PTSD currently have enough evidence to justify clinical use. The intervention with the most evidence at this time is hydrocortisone, which has shown promise in two RCTs of patients who were critically ill or undergoing major surgery as well as two RCTs in adult trauma survivors. Based on prospective clinical studies, the evidence for SSRIs in preventing PTSD is equivocal based on two small studies, while propranolol and benzodiazepines have not found support. Observational studies have found a link between opioid administration after trauma and lower rates of subsequent PTSD, although this has not been tested in RCTs. One small observational study found evidence of a link between albuterol administration and decreased PTSD, while findings on the effect of ketamine have been mixed. Preclinical research has identified a number of promising strategies for PTSD prevention, including modulators of the stress response and strategies to disrupt traumatic memory consolidation. 


\section{SUMMARY AND CONCLUSIONS}

Overall, evidence for preventive interventions for ASD and PTSD is scarce. Some interventions have not been supported by the evidence, while others have shown promise but require further testing. Approaches to prevention of ASD and PTSD have taken a number of different avenues. While outside the scope of this review, public policy and public health interventions to reduce violence, traumatic injuries, and other traumatic events have a major role to play in reducing the incidence of ASD and PTSD. There may also be opportunities to bolster resilience in vulnerable individuals before trauma, although research in this area is lacking. To date, most clinical research has focused on secondary prevention of PTSD.

Optimal targeting of secondary prevention interventions remains largely unexplored. Most approaches have either targeted all individuals experiencing a trauma or have targeted individuals with acute stress symptoms. There is a growing body of research on predictors of PTSD (based on pretrauma factors and reactions after trauma), which may be useful in directing interventions to individuals at highest risk. However, the clinical utility of currently known risk factors for this purpose has not been tested.

The most studied psychosocial interventions to prevent PTSD are single-session PD approaches and multi-session CBT-based approaches. Evidence indicates that PD is not an effective intervention to prevent PTSD. On balance, research suggests that CBT is effective when ASD symptoms are present, but its usefulness in the absence of posttraumatic symptoms is unclear. Several other psychosocial interventions, such as a collaborative care approach, have shown promise but require more study.

Only a handful of medications have been tested in prospective studies, and many results have been disappointing. There is some evidence that hydrocortisone may be effective in preventing PTSD, but appropriate patient selection needs to be clarified in terms of risk/benefit tradeoffs. More research on this approach is needed. Other medications, such as propranolol, are not supported by current evidence. Observational studies have found a link between opioid administration and lower rates of subsequent PTSD, but opioids have not been tested in RCTs. If opioids are found to be effective, then risks and benefits will need to be considered when determining appropriate patient selection. A number of preclinical studies have identified medications that modulate the stress response, fear conditioning, and memory consolidation and appear to prevent PTSD-like behaviors in animal models. These promising strategies have not yet been tested in clinical trials.

\section{FUTURE RESEARCH DIRECTIONS}

The promise of preventive interventions for stress-related disorders has not yet been fulfilled. The clinical benefits of prevention can most likely be achieved by pursuing a translational research strategy in which the design of clinical studies is rigorously based on neurobiological discoveries. It is crucial to direct resources to adequately test emerging interventions so that preclinical findings can enhance the lives of trauma survivors and alleviate the public health burden of stress-related disorders.

Proper patient selection will be an important consideration for any preventive interventions. Most studies to date have directed interventions either to all trauma survivors or to those with acute stress symptoms. However, advancing understanding of the factors underlying development of PTSD may result in more sophisticated targeting strategies. While a number of pretrauma factors as well as aspects of early response to trauma have emerged as risk factors for PTSD, most of these factors have not yet been shown to be clinically relevant for targeting interventions. It is possible that a clinical prediction tool which combines a number of factors could be accurate enough for this purpose, and development of such a tool could represent a useful research direction. In addition to predicting PTSD, research on individual variability in response to interventions could also be helpful in personalizing the treatment of trauma survivors and optimizing outcomes. The ability to select patients on a basis other than acute stress symptoms may also allow earlier intervention (hours rather than days or weeks after trauma), which may improve outcomes. New tools to aid in the development of alternative classification systems and dimensional approaches to PTSD may prove useful in this regard. These include confirmatory factor analysis, latent class analysis, and machine learning methods. Approaches to identify latent trajectories may also help clarify which individuals benefit most from preventive interventions.

In addition to identifying neural targets for intervention, preclinical research can also help identify the ideal timing of interventions. It is notable that many preclinical studies administer interventions within hours or days after stressor exposure, while some clinical studies do not intervene until weeks have elapsed after a trauma. A fuller understanding of processes involved in the development of PTSD including the stress response, fear conditioning, and memory consolidation could help guide the timing of different interventions. Again, using the principles of translational research by tying clinical study design to preclinical findings can ensure the best use of resources when testing clinical interventions.

Other aspects of clinical study design also require attention. In addition to adequate sample sizes, randomization, adequate controls, and other principles of rigorous design, outcome measurement is an important consideration. The studies reviewed here vary considerably in how outcomes are measured, whether PTSD incidence or level of symptoms is the primary outcome, and time to follow-up. This last point is particularly important given that some interventions may have short-term benefits that do not persist. Long-term follow-up studies are important to ensure that preventive interventions decrease PTSD beyond the first few weeks or months after trauma.

It is difficult to predict which types of interventions will be most effective in preventing stress-related disorders, and 
multiple avenues should be pursued. This includes primary prevention strategies to enhance resilience in those at highest risk for trauma as well as secondary interventions administered after trauma. Likewise, both psychosocial and somatic approaches should be pursued. Each of these approaches may have advantages and disadvantages. Safe and effective medications may be easier to disseminate widely when compared with psychosocial interventions, and both types of interventions may differ in terms of risk profiles. As always, risk and benefits of intervention must be carefully considered before any intervention is used in a clinical setting.

Overall, the potential benefits of prevention of stress related disorders strongly argue for more research attention to this area. With sufficient resources and attention to the principles of translational research, there is reason to believe that successful interventions can be developed. Advances in neurobiological understanding of responses to trauma raise the exciting prospect that clinicians can intervene to prevent the extensive suffering and disability of PTSD and related disorders before they begin.

\section{FUNDING AND DISCLOSURE}

Dr Stein reports consulting income in the past 3 years from Janssen Pharmaceutica, Pfizer, and Tonix Pharmaceuticals, and income for editorial work for Biological Psychiatry and UpToDate. Drs. Howlett and Stein will be receiving funds through their institution to participate in an RCT of a Janssen drug for an indication other than PTSD.

\section{REFERENCES}

Adamec R, Fougere D, Risbrough V (2010). CRF receptor blockade prevents initiation and consolidation of stress effects on affect in the predator stress model of PTSD. Int J Neuropsychopharmacol 13: 747-757.

Adler AB, Bliese PD, McGurk D, Hoge CW, Castro CA (2011). Battlemind debriefing and Battlemind training as early interventions with soldiers returning from Iraq. Sport Exerc Performance Psychol 1: 66-83.

Agorastos A, Marmar CR, Otte C (2011). Immediate and early behavioral interventions for the prevention of acute and posttraumatic stress disorder. Curr Opin Psychiatry 24: 526-532.

Almli LM, Fani N, Smith AK, Ressler KJ (2014). Genetic approaches to understanding post-traumatic stress disorder. Int $\mathrm{J}$ Neuropsychopharmacol 17: 355-370.

American Psychiatric Association (2000). Diagnostic and Statistical Manual-Text Revision (DSM-IV-TRim, 2000). American Psychiatric Association: Washington, DC

American Psychiatric Association (2013). Diagnostic and Statistical Manual of Mental Disorders: DSM-V. American Psychiatric Association: Washington, DC.

Andero R, Brothers SP, Jovanovic T, Chen YT, Salah-Uddin H, Cameron M et al (2013). Amygdala-dependent fear is regulated by Oprl1 in mice and humans with PTSD. Sci Transl Med 5: 188ra173.

Ashton $\mathrm{H}$ (2005). The diagnosis and management of benzodiazepine dependence. Curr Opin Psychiatry 18: 249-255.

Barbee JG (1993). Memory, benzodiazepines, and anxiety: integration of theoretical and clinical perspectives. J Clin Psychiatry 54(Suppl) 86-97; discussion 98-101.

Berkowitz SJ, Stover CS, Marans SR (2011). The child and family traumatic stress intervention: secondary prevention for youth at risk of developing PTSD. J Child Psychol Psychiatry 52: 676-685.

Bremner J, Davis M, Southwick S, Krystal J, Charney D (1993). Neurobiology of posttraumatic stress disorder. Rev Psychiatry 12: 183-204.

Breslau N (2012). Epidemiology of posttraumatic stress disorder in adults. In: Beck JG, Sloan DM (eds), The Oxford Handbook of Traumatic Stress Disorders. Oxford University Press: New York, pp 84-97.
Brewin CR, Andrews B, Rose S, Kirk M (2014). Acute stress disorder and posttraumatic stress disorder in victims of violent crime. Am J Psychiatry 156: 360-366.

Bryant RA (2003). Early predictors of posttraumatic stress disorder. Biol Psychiatry 53: 789-795.

Bryant RA (2007). Early intervention for post-traumatic stress disorder. Early Interv Psychiatry 1: 19-26.

Bryant RA (2011). Acute stress disorder as a predictor of posttraumatic stress disorder: a systematic review. J Clin Psychiatry 72: 233-239.

Bryant RA, Creamer M, O'Donnell M, Silove D, McFarlane AC (2008). A multisite study of initial respiration rate and heart rate as predictors of posttraumatic stress disorder. J Clin Psychiatry 69: 1694-1701.

Bryant RA, Creamer M, O'Donnell M, Silove D, McFarlane AC (2009). A study of the protective function of acute morphine administration on subsequent posttraumatic stress disorder. Biol Psychiatry 65: 438-440.

Bryant RA, Creamer M, O'Donnell M, Silove D, McFarlane AC, Forbes D (2015). A comparison of the capacity of DSM-IV and DSM-5 acute stress disorder definitions to predict posttraumatic stress disorder and related disorders. J Clin Psychiatry 76: 391-397.

Bryant RA, Nickerson A, Creamer M, O'Donnell M, Forbes D, Galatzer-Levy I et al (2015). Trajectory of post-traumatic stress following traumatic injury: 6-year follow-up. Br J Psychiatry 206: 417-423.

Cahill L, Pham CA, Setlow B (2000). Impaired memory consolidation in rats produced with $\beta$-adrenergic blockade. Neurobiol Learn Mem 74: 259-266.

Caplan G (1964). Principles of preventive psychiatry. Basic Books: New York.

Cohen H, Kaplan Z, Kozlovsky N, Gidron Y, Matar M, Zohar J (2010). Hippocampal microinfusion of oxytocin attenuates the behavioural response to stress by means of dynamic interplay with the glucocorticoid-catecholamine responses. J Neuroendocrinol 22: 889-904.

Cohen H, Kaplan Z, Matar MA, Loewenthal U, Kozlovsky N, Zohar J (2006). Anisomycin, a protein synthesis inhibitor, disrupts traumatic memory consolidation and attenuates posttraumatic stress response in rats. Biol Psychiatry 60: $767-776$.

Cohen H, Matar MA, Buskila D, Kaplan Z, Zohar J (2008). Early post-stressor intervention with high-dose corticosterone attenuates posttraumatic stress response in an animal model of posttraumatic stress disorder. Biol Psychiatry 64: 708-717.

Cohen S, Kozlovsky N, Matar MA, Kaplan Z, Zohar J, Cohen H (2012). Postexposure sleep deprivation facilitates correctly timed interactions between glucocorticoid and adrenergic systems, which attenuate traumatic stress responses. Neuropsychopharmacology 37: 2388-2404.

Commission on Chronic Illness (1957). Chronic Illness in the United States. Harvard University Press: Cambridge, MA, Vol. 1.

Delahanty DL, Gabert-Quillen C, Ostrowski SA, Nugent NR, Fischer B, Morris A et al (2013). The efficacy of initial hydrocortisone administration at preventing posttraumatic distress in adult trauma patients: a randomized trial. CNS Spectr 18: 103-111.

Delahanty DL, Raimonde AJ, Spoonster E (2000). Initial posttraumatic urinary cortisol levels predict subsequent PTSD symptoms in motor vehicle accident victims. Biol Psychiatry 48: 940-947.

DiGangi JA, Gomez D, Mendoza L, Jason LA, Keys CB, Koenen KC (2013). Pretrauma risk factors for posttraumatic stress disorder: a systematic review of the literature. Clin Psychol Rev 33: 728-744.

Dunmore E, Clark DM, Ehlers A (2001). A prospective investigation of the role of cognitive factors in persistent posttraumatic stress disorder (PTSD) after physical or sexual assault. Behav Res Ther 39: 1063-1084.

Elías PC, Sagua D, Alvarez EO (2004). Chronic aerial exposure to glucorticoids or beta-agonists affects avoidance learning and exploratory motivation in rats. Behav Brain Res 149: 95-105.

Forbes D, Elhai JD, Miller MW, Creamer M (2010). Internalizing and externalizing classes in posttraumatic stress disorder: a latent class analysis. J Trauma Stress 23: 340-349.

Forbes D, Lockwood E, Creamer M, Bryant RA, McFarlane AC, Silove D et al (2015a). Latent structure of the proposed ICD-11 post-traumatic stress disorder symptoms: implications for the diagnostic algorithm. Br J Psychiatry 206: 245-251.

Forbes D, Nickerson A, Alkemade N, Bryant R, Creamer M, Silove D et al (2015b). Longitudinal analysis of latent classes of psychopathology and patterns of class migration in survivors of severe injury. J Clin Psychiatry; e-pub ahead of print 14 April 2015. doi:10.4088/JCP.14m09075.

Forneris CA, Gartlehner G, Brownley KA, Gaynes BN, Sonis J, Coker-Schwimmer E et al (2013). Interventions to prevent post-traumatic stress disorder: a systematic review. Am J Prev Med 44: 635-650.

Galatzer-Levy IR, Ankri Y, Freedman S, Israeli-Shalev Y, Roitman P, Gilad M et al (2013). Early PTSD symptom trajectories: Persistence, recovery, and response to 
treatment: results from the Jerusalem Trauma Outreach and Prevention Study (J-TOPS). PLoS One 8: e70084.

Galatzer-Levy IR, Karstoft K-I, Statnikov A, Shalev AY (2014). Quantitative forecasting of PTSD from early trauma responses: a Machine Learning application. J Psychiatr Res 59: 68-76.

Galatzer-Levy IR, Nickerson A, Litz BT, Marmar CR (2013). Patterns of lifetime PTSD comorbidity: a latent class analysis. Depress Anxiety 30: 489-496.

Ganon-Elazar E, Akirav I (2012). Cannabinoids prevent the development of behavioral and endocrine alterations in a rat model of intense stress. Neuropsychopharmacology 37: 456-466.

Gelpin E, Bonne O, Peri T, Brandes D, Shalev AY (1996). Treatment of recent trauma survivors with benzodiazepines: a prospective study. J Clin Psychiatry 57: 390-394.

Gidron Y, Gal R, Freedman S, Twiser I, Lauden A, Snir Y et al (2001). Translating research findings to PTSD prevention: Results of a randomized-controlled pilot study. J Trauma Stress 14: 773-780.

Gidron Y, Gal R, Givati G, Lauden A, Snir Y, Benjamin J (2007). Interactive effects of memory structuring and gender in preventing posttraumatic stress symptoms. J Nerv Ment Dis 195: 179-182.

Good AJ, Westbrook R (1995). Effects of a microinjection of morphine into the amygdala on the acquisition and expression of conditioned fear and hypoalgesia in rats. Behav Neurosci 109: 631

Gordon RS Jr (1983). An operational classification of disease prevention. Public Health Rep 98: 107.

Harvey AG, Bryant RA, Tarrier N (2003). Cognitive behaviour therapy for posttraumatic stress disorder. Clin Psychol Rev 23: 501-522.

Hoge EA, Worthington JJ, Nagurney JT, Chang Y, Kay EB, Feterowski CM et al (2012). Effect of acute posttrauma propranolol on PTSD outcome and physiological responses during script-driven imagery. CNS Neurosci Ther 18: 21-27.

Holbrook TL, Galarneau MR, Dye JL, Quinn K, Dougherty AL (2010). Morphine use after combat injury in Iraq and post-traumatic stress disorder. N Engl J Med 362: 110-117.

Hourani LL, Council CL, Hubal RC, Strange LB (2011). Approaches to the primary prevention of posttraumatic stress disorder in the military: a review of the stress control literature. Mil Med 176: 721-730.

Huang Z-I, Liu R, Bai X-y, Zhao G, Song J-k, Wu S et al (2014). Protective effects of the novel adenosine derivative WS0701 in a mouse model of posttraumatic stress disorder. Acta Pharmacol Sin 35: 24-32.

Insel T, Cuthbert B, Garvey M, Heinssen R, Pine DS, Quinn K et al (2010). Research domain criteria (RDoC): toward a new classification framework for research on mental disorders. Am J Psychiatry 167: 748-751.

Jonas DE, Cusack K, Forneris CA, Wilkins TM, Sonis J, Middleton JC et al (2013). Psychological and Pharmacological Treatments for Adults with Posttraumatic Stress Disorder (PTSD). Agency for Healthcare Research and Quality, US Department of Health and Human Services: Rockville, MD.

Juven-Wetzler A, Cohen H, Kaplan Z, Kohen A, Porat O, Zohar J (2014). Immediate ketamine treatment does not prevent posttraumatic stress responses in an animal model for PTSD. Eur Neuropsychopharmacol 24: 469-479.

Kawakita E, Hashimoto M, Shido O (2006). Docosahexaenoic acid promotes neurogenesis in vitro and in vivo. Neuroscience 139: 991-997.

Kearns MC, Ressler KJ, Zatzick D, Rothbaum BO (2012). Early interventions for PTSD: a review. Depres Anxiety 29: 833-842.

Kessler RC (2000). Posttraumatic stress disorder: the burden to the individual and to society. J Clin Psychiatry 61: 4-14.

Kessler RC, Berglund P, Demler O, Jin R, Merikangas KR, Walters EE (2005). Lifetime prevalence and age-of-onset distributions of DSM-IV disorders in the National Comorbidity Survey Replication. Arch Gen Psychiatry 62: 593-602.

Kessler RC, Rose S, Koenen KC, Karam EG, Stang PE, Stein DJ et al (2014). How well can post-traumatic stress disorder be predicted from pre-trauma risk factors? An exploratory study in the WHO World Mental Health Surveys. World Psychiatry 13: 265-274.

King DW, Leskin GA, King LA, Weathers FW (1998). Confirmatory factor analysis of the clinician-administered PTSD scale: evidence for the dimensionality of posttraumatic stress disorder. Psychol Assess 10: 90.

Kitamura T, Saitoh Y, Takashima N, Murayama A, Niibori Y, Ageta H et al (2009). Adult neurogenesis modulates the hippocampus-dependent period of associative fear memory. Cell 139: 814-827.

Kobayashi I, Sledjeski E, Fallon W, Spoonster E, Riccio D, Delahanty D (2011). Effects of early albuterol (salbutamol) administration on the development of posttraumatic stress symptoms. Psychiatry Res185: 296-298.

Kraus JF, Peek-Asa C, Vimalachandra D (1998). Injury control: the public health approach. In: Wallace RB (ed), Maxcy-Rosenau-Last Public Health and Preventive Medicine, 14 edn. Appleton \& Lange: Stamford, CT.
Kripke DF (2009). Do hypnotics cause death and cancer? The burden of proof. Sleep Med 10: 275-276.

Lewitus GM, Cohen H, Schwartz M (2008). Reducing post-traumatic anxiety by immunization. Brain Behav Immun 22: 1108-1114.

Litz BT, Gray MJ, Bryant RA, Adler AB (2002). Early intervention for trauma: current status and future directions. Clin Psychol Sci Pract 9: 112-134.

Logue MW, Amstadter AB, Baker DG, Duncan L, Koenen KC, Liberzon I et al (2015). The Psychiatric Genomics Consortium Posttraumatic Stress Disorder Workgroup: posttraumatic stress disorder enters the age of large-scale genomic collaboration. Neuropsychopharmacology 40: 2287-2297.

Lund BC, Bernardy NC, Alexander B, Friedman MJ (2012). Declining benzodiazepine use in veterans with posttraumatic stress disorder. J Clin Psychiatry 73 292-296.

Matsuoka Y, Nishi D, Yonemoto N, Hamazaki K, Hamazaki T, Hashimoto K (2011). Potential role of brain-derived neurotrophic factor in omega-3 fatty acid supplementation to prevent posttraumatic distress after accidental injury: an open-label pilot study. Psychother Psychosom 80: 310.

McFarlane AC, Atchison M, Yehuda R (1997). The acute stress response following motor vehicle accidents and its relation to PTSD. Ann NY Acad Sci 821: 437-441.

McGhee LL, Maani CV, Garza TH, Gaylord KM, Black IH (2008). The correlation between ketamine and posttraumatic stress disorder in burned service members. J Trauma 64: S195-S199.

McGhee LL, Maani CV, Garza TH, Slater TM, Petz LN, Fowler M (2014). The intraoperative administration of ketamine to burned US Service members does not increase the incidence of post-traumatic stress disorder. Mil Med 179: 41-46.

Mellman M, Thomas A, Bustamante V, David D, Fins Al (2002). Hypnotic medication in the aftermath of trauma. J Clin Psychiatry 63: 1183

Mitchell J, Everly G (1993). Critical Incident Stress Debriefing: An Operations Manual for the Prevention of Trauma Among Emergency Service and Disaster Workers. Chevron: Baltimore, MD.

Mulligan K, Fear NT, Jones N, Alvarez H, Hull L, Naumann U et al (2012). Postdeployment Battlemind training for the UK armed forces: a cluster randomized controlled trial. J Consult Clin Psychol 80: 331.

Nievergelt CM, Maihofer AX, Mustapic M, Yurgil KA, Schork NJ, Miller MW et al (2015). Genomic predictors of combat stress vulnerability and resilience in US Marines: a genome-wide association study across multiple ancestries implicates PRTFDC1 as a potential PTSD gene. Psychoneuroendocrinology 51: 459-471.

Nugent NR, Christopher NC, Crow JP, Browne L, Ostrowski S, Delahanty DL (2010). The efficacy of early propranolol administration at reducing PTSD symptoms in pediatric injury patients: a pilot study. J Trauma Stress 23: 282-287.

Pitman RK, Sanders KM, Zusman RM, Healy AR, Cheema F, Lasko NB et al (2002). Pilot study of secondary prevention of posttraumatic stress disorder with propranolol. Biol Psychiatry 51: 189-192.

Price M, Kearns M, Houry D, Rothbaum BO (2014). Emergency department predictors of posttraumatic stress reduction for trauma-exposed individuals with and without an early intervention. J Consult Clin Psychol 82: 336

Rasmussen KG (2015). Has psychiatry tamed the "Ketamine Tiger?" considerations on its use for depression and anxiety. Prog Neuropsychopharmacol Biol Psychiatry pii: S0278-5846(15)00003-2.

Resnick H, Acierno R, Waldrop AE, King L, King D, Danielson C et al (2007). Randomized controlled evaluation of an early intervention to prevent post-rape psychopathology. Behav Res Ther 45: 2432-2447.

Riemann D, Nissen C, Palagini L, Otte A, Perlis ML, Spiegelhalder K (2015). The neurobiology, investigation, and treatment of chronic insomnia. Lancet Neurol 14 547-558

Roberts NP, Kitchiner NJ, Kenardy J, Bisson JI (2009). Systematic review and metaanalysis of multiple-session early interventions following traumatic events. Am J Psychiatry 166: 293-301.

Roberts NP, Kitchiner NJ, Kenardy J, Bisson Jl (2010). Early psychological interventions to treat acute traumatic stress symptoms. Cochrane Database Syst Rev CD007944

Rose G (1992). Strategy of Preventive Medicine. Oxford University Press: Oxford.

Rose S, Bisson J, Churchill R, Wessely S (2002). Psychological debriefing for preventing post traumatic stress disorder (PTSD). Cochrane Database Syst Rev CD000560.

Rose S, Brewin CR, Andrews B, Kirk M (1999). A randomized controlled trial of individual psychological debriefing for victims of violent crime. Psychol Med 29: 793-799.

Rosenberg ML, Mercy JA, Hammond WR (1998). Assaultive violence. In: Wallace RB(ed), Maxcy-Rosenau-Last Public Health and Preventive Medicine, 14 edn. Appleton \& Lange: Stamford, CT.

Rothbaum BO, Kearns MC, Price M, Malcoun E, Davis M, Ressler KJ et al (2012). Early intervention may prevent the development of posttraumatic stress disorder: 
a randomized pilot civilian study with modified prolonged exposure. Biol Psychiatry 72: 957-963.

Rothbaum BO, Kearns MC, Reiser ME, Davis MJS, Kerley MKA, Rothbaum MAO et al (2014). Early intervention following trauma may mitigate genetic risk for PTSD in civilians: a pilot prospective emergency department study. J Clin Psychiatry 75: 1380.

Sabban EL, Serova LI, Alaluf LG, Laukova M, Peddu C (2014). Comparative effects of intranasal neuropeptide $\mathrm{Y}$ and HS014 in preventing anxiety and depressive-like behavior elicited by single prolonged stress. Behav Brain Res pii: S0166-4328(14)00841-9.

Saxe G, Stoddard F, Courtney D, Cunningham K, Chawla N, Sheridan R et al (2001). Relationship between acute morphine and the course of PTSD in children with burns. J Am Acad Child Adolesc Psychiatry 40: 915-921.

Schelling G, Briegel J, Roozendaal B, Stoll C, Rothenhäusler H-B, Kapfhammer H-P (2001). The effect of stress doses of hydrocortisone during septic shock on posttraumatic stress disorder in survivors. Biol Psychiatry 50: 978-985.

Schelling G, Kilger E, Roozendaal B, Dominique J-F, Briegel J, Dagge A et al (2004). Stress doses of hydrocortisone, traumatic memories, and symptoms of posttraumatic stress disorder in patients after cardiac surgery: a randomized study. Biol Psychiatry 55: 627-633.

Schelling G, Stoll C, Kapfhammer H-P, Rothenhäusler H-B, Krauseneck T, Durst K et al (1999). The effect of stress doses of hydrocortisone during septic shock on posttraumatic stress disorder and health-related quality of life in survivors. Crit Care Med 27: 2678-2683.

Schönenberg M, Reichwald U, Domes G, Badke A, Hautzinger M (2005). Effects of peritraumatic ketamine medication on early and sustained posttraumatic stress symptoms in moderately injured accident victims. Psychopharmacology 182: 420-425.

Shalev AY, Ankri Y, Israeli-Shalev Y, Peleg T, Adessky R, Freedman S (2012). Prevention of posttraumatic stress disorder by early treatment: results from the Jerusalem Trauma Outreach and Prevention Study. Arch Gen Psychiatry 69: 166-176.

Sharp S, Thomas C, Rosenberg L, Rosenberg M, Meyer W III (2010). Propranolol does not reduce risk for acute stress disorder in pediatric burn trauma. J Trauma Acute Care Surg 68: 193-197.

Sharpley J, Fear N, Greenberg N, Jones M, Wessely S (2008). Pre-deployment stress briefing: does it have an effect? Occup Med 58: 30-34.

Simms LJ, Watson D, Doebbelling BN (2002). Confirmatory factor analyses of posttraumatic stress symptoms in deployed and nondeployed veterans of the Gulf War. J Abnorm Psychol 111: 637.

Skeffington PM, Rees CS, Kane R (2013). The primary prevention of PTSD: a systematic review. J Trauma Dissociation 14: 404-422.

Southwick SM, Bremner JD, Rasmusson A, Morgan CA, Arnsten A, Charney DS (1999). Role of norepinephrine in the pathophysiology and treatment of posttraumatic stress disorder. Biol Psychiatry 46: 1192-1204.
Steenkamp MM, Nickerson A, Maguen S, Dickstein BD, Nash WP, Litz BT (2012). Latent classes of PTSD symptoms in Vietnam veterans. Behav Modif 36: 857-874.

Stein MB, Kerridge C, Dimsdale JE, Hoyt DB (2007). Pharmacotherapy to prevent PTSD: Results from a randomized controlled proof-of-concept trial in physically injured patients. J Trauma Stress 20: 923-932.

Stein MB, Lang AJ (2013). What's impeding post-traumatic stress disorder prevention? Am J Prev Med 44: 692-693.

Stoddard FJ Jr, Luthra R, Sorrentino EA, Saxe GN, Drake J, Chang Y et al (2011). A randomized controlled trial of sertraline to prevent posttraumatic stress disorder in burned children. J Child Adolesc Psychopharmacol 21: 469-477.

Stoddard FJ Jr, Sorrentino EA, Ceranoglu TA, Saxe G, Murphy JM, Drake JE et al (2009). Preliminary evidence for the effects of morphine on posttraumatic stress disorder symptoms in one-to four-year-olds with burns. J Burn Care Res 30: 836-843.

Treggiari MM, Romand J-A, Yanez ND, Deem SA, Goldberg J, Hudson L et al (2009). Randomized trial of light versus deep sedation on mental health after critical illness. Crit Care Med 37: 2527-2534.

Vaiva G, Ducrocq F, Jezequel K, Averland B, Lestavel P, Brunet A et al (2003). Immediate treatment with propranolol decreases posttraumatic stress disorder two months after trauma. Biol Psychiatry 54: 947-949.

Vaiva G, Thomas P, Ducrocq F. Fontaine M, Boss V, Devos P et al (2004). Low posttrauma GABA plasma levels as a predictive factor in the development of acute posttraumatic stress disorder. Biol Psychiatry 55: 250-254.

Wessely S, Bryant RA, Greenberg N, Earnshaw M, Sharpley J, Hughes JH (2008). Does psychoeducation help prevent post traumatic psychological distress? Psychiatry 71: 287-302.

Wolf EJ, Miller MW, Reardon AF, Ryabchenko KA, Castillo D, Freund R (2012). A latent class analysis of dissociation and posttraumatic stress disorder: evidence for a dissociative subtype. Arch Gen Psychiatry 69: 698-705.

Zatzick D, Jurkovich G, Rivara FP, Russo J, Wagner A, Wang J et al (2013). A randomized stepped care intervention trial targeting posttraumatic stress disorder for surgically hospitalized injury survivors. Ann Surg 257: 390

Zelazny K, Simms LJ (2015). Confirmatory factor analyses of DSM-5 posttraumatic stress disorder symptoms in psychiatric samples differing in criterion a status. J Anxiety Disord 34: 15-23.

Zhang L-M, Zhou W-W, Ji Y-J, Li Y, Zhao N, Chen H-X et al (2014). Anxiolytic effects of ketamine in animal models of posttraumatic stress disorder. Psychopharmacology 232: 663-672.

Zohar J, Yahalom H, Kozlovsky N, Cwikel-Hamzany S, Matar MA, Kaplan Z et al (2011). High dose hydrocortisone immediately after trauma may alter the trajectory of PTSD: interplay between clinical and animal studies. Eur Neuropsychopharmacol 21: 796-809.

Zoladz PR, Diamond DM (2013). Current status on behavioral and biological markers of PTSD: a search for clarity in a conflicting literature. Neurosci Biobehav Rev 37: 860-895. 